\title{
Evaluación de la calidad del agua superficial utilizando el índice de calidad del agua (ICA). Caso de estudio: Cuenca del Río Guarapiche, Monagas, Venezuela
}

\author{
Assessment of surface water quality using water quality index. (WQI) Case study: Guarapiche \\ River Basin, Monagas, Venezuela
}

\author{
José Alexander Gil-Marín ${ }^{*}$, Celeidys Vizcaino ${ }^{1}$, Nelson José Montaño-Mata ${ }^{1}$
}

* Autor de correspondencia

\begin{abstract}
Resumen
El objetivo de esta investigación fue evaluar el índice de calidad del agua (ICA) en el río Guarapiche, estado Monagas, Venezuela, medido por el método del índice aritmético ponderado. Para ello, se evaluó catorce parámetros de calidad del agua (temperatura, $\mathrm{pH}$, dureza, $\mathrm{CE}$, nitrato, nitritos, sulfato, cloruros, $\mathrm{OD}, \mathrm{Fe}, \mathrm{Mn}, \mathrm{Ni}, \mathrm{K}, \mathrm{y} \mathrm{CF}$ ). El peso relativo asignado a cada parámetro varió de uno a cuatro basado sobre la importancia del parámetro para la vida acuática. Los valores de ICA van desde 44,38 (muestreo estación San Félix) a 363,69 (estación de muestreo Palmonagas). El impacto de varias actividades antropogénicas fue evidente en algunos parámetro como $\mathrm{Mn}, \mathrm{NO}_{3}, \mathrm{NO}_{2}$, y $\mathrm{CF}$. Se sugiere que el monitoreo del río es necesario para una gestión adecuada. La aplicación del ICA se recomienda como una herramienta muy útil que permite al público y a los responsables de la toma de decisiones, evaluar la calidad del agua de los ríos en Venezuela.
\end{abstract}

Palabras clave: índice de calidad del agua; recursos hídricos; río Guarapiche; antropogénicas; Venezuela.

\begin{abstract}
The objective of this research was to evaluate the water quality index (ICA) in the Guarapiche River, Monagas State, Venezuela, measured by the weighted arithmetic index method. For this, fourteen parameters of water quality (temperature, $\mathrm{pH}$, hardness, $\mathrm{EC}$, nitrate, nitrites, sulfate, chlorides, $\mathrm{OD}, \mathrm{Fe}, \mathrm{Mn}, \mathrm{Ni}, \mathrm{K}$, and $\mathrm{CF}$ ) were evaluated. The relative weight assigned to each parameter varied from one to four based on the importance of the parameter for aquatic life. The ICA values range from 44,38 (San Felix station sampling) to 363,69 (Palmonagas sampling station). The impact of several anthropogenic activities was evident in some parameters, such as $\mathrm{Mn}, \mathrm{NO}_{3}, \mathrm{NO}_{2}$, and CF. It is suggested that river monitoring is necessary for proper management. The application of the ICA is recommended as a very useful tool that allows the public and decision makers to evaluate the quality of the water in rivers in Venezuela.
\end{abstract}

Keywords: water quality index; water resources; Guarapiche river; anthropogenic; Venezuela.

\section{Introducción}

El aumento de la población causa un aumento de la necesidad de los recursos naturales. Las consecuencias directas son para la cantidad y calidad del agua dulce disponible para el consumo humano. Los índices de calidad del agua son un componente importante del manejo global integrado de los recursos hídricos (Lekshmiprasad y Mophin, 2017). Cuando no se cumplen con los estándares de los índices de calidad del agua, los usuarios deben pagar un costo adicional por el tratamiento del agua para disminuir el riesgo de daño o pérdida. Los principales esfuerzos y costos involucrados en la gestión del agua están dedicados a la protección y gestión de su calidad (J). Los conflictos entre los diversos usuarios del agua están relacionadas con la calidad y la cantidad de la misma (Sánchez et al., 2007). Los cuerpos de aguas naturales pueden servir para muchos usos, incluido el transporte y la asimilación de desechos. Pero a medida que los cuerpos de agua integran estos desechos, su calidad cambia. Las necesidades humanas más fundamentales del agua son para beber, cocinar y saneamiento personal. Para satisfacer estas necesidades, la calidad del agua utilizada no debe representar ningún riesgo para la salud humana. La calidad del agua en la naturaleza afecta la condición de los ecosistemas afectando la vida de los eres vivos. Al mismo tiempo, los seres humanos utilizan cuerpos de agua como medios convenientes para la eliminación de aguas residuales domésticas, industriales y agrícolas; que degrada la calidad de esos cuerpos de agua. Una gestión de los recursos hídricos involucra el control y la calidad del agua (Bhattacharya et al., 2012). Se han desarrollado varíos modelos para ayudar a predecir los impactos en la calidad del agua. Así como elaborar estrategias y prácticas alternativas en el manejo del suelos y del agua (Cude, 2001). 
Todos los organismos vivos requieren agua de suficiente cantidad y calidad para sobrevivir, aunque diferentes especies acuáticas pueden tolerar diferentes niveles de calidad del agua (Lekshmiprasad y Mophin, 2017). Sin embargo, en el mundo desarrollado, no es seguro beber agua de fuentes superficiales naturales o subterráneas; generalmente necesitan ser tratados antes de que sean aptos para el consumo humano. El tratamiento no es una opción práctica para el recreo, el baño o para mantener la salubridad de los peces, mariscos y otros organismos que se encuentran en los ecosistemas acuáticos naturales (Smith, 1990). Por lo tanto, las normas que establecen niveles mínimos aceptables de calidad se fijan para la mayoría de las aguas ambientales. Otros usos tienen también sus propios estándares. El agua de riego no debe tener niveles de salinidad por encima de $3 \mathrm{dS} / \mathrm{m}$, ni contener sustancias tóxicas que puedan ser absorbidas por las plantas. Los estándares de calidad del agua para la industria pueden ser más difíciles de establecer, y dependen por supuesto de los procesos industriales involucrados. Debido a las dificultades relacionadas con los recursos hídricos, es importante desarrollar monitoreo, investigación y manejo para garantizar su gestión efectiva. El río Guarapiche es la principal fuente proveedora de agua en el estado Monagas, Venezuela. Es uno de los afluentes con mayor potencial de uso para diferentes actividades, entre las cuales resaltan la agroindustrial, la agrícola, la petrolera, recreacional y la de uso doméstico. Esta fuente, en los últimos años, se ha convertido en un vertedero de desechos de las poblaciones adyacentes y establecimientos industriales. Aparte de eso, el derrame de petróleo en los últimos años ha afectado a la población de peces. Otra fuente de contaminación es la escorrentía de los campos agrícolas en las áreas adyacentes. Los fertilizantes y pesticidas químicos producen la eutrofización del río. Las pequeñas industrias contribuyen con la contaminación. Para examinar los impactos de estas actividades, se necesita una evaluación de la calidad del agua. Este estudio presenta al índice de calidad del agua (ICA), el cual es un tipo de índice ambiental que puede ser usado como marco de referencia único para comunicar información sobre la calidad del ambiente afectado y para evaluar la vulnerabilidad o la susceptibilidad del agua a la contaminación (Canter,1996). También, se puede considerar como una forma de agrupación simplificada de algunos constituyentes que indican el deterioro en la calidad de agua. El ICA posibilita la evaluación de los constituyentes que afectan la calidad del agua para sus diferentes usos. Esta evaluación nos sirve como una manera de comunicar y representar la calidad del agua en los cuerpos de agua. Además, permite la comparación entre diversos ríos o en entre diferentes localizaciones para un mismo río.

El ICA utiliza la documentación de calidad del agua y se soporta en la variación de las políticas, que están enmarcadas por varias agencias de monitoreo ambiental (Kung et al., 1992). El procedimiento individual de la variable calidad del agua no es fácil de entender para el público. Por esa razón, el ICA sintetiza la mayor información en un solo valor para expresar los datos en una forma simple y lógica. La recolección de datos, de varias fuentes, permite desarrollar un estado completo del cuerpo de agua. Esto aumenta la capacidad de comprensión de los problemas de calidad del agua tanto por parte de los responsables políticos, como del público y demás usuarios de los recursos hídricos. El río Guarapiche se somete a múltiples prácticas para el suministro de agua comunitaria, riego, limpieza, aseo, eliminación de aguas residuales y efluentes industriales. El presente trabajo tiene como objetivo desarrollar un marco para descubrir el escenario de calidad del agua del río Guarapiche y encontrar las soluciones para el manejo adecuado de esta fuente de agua dulce a través del índice de calidad del agua.

\section{Materiales y métodos}

El área de estudio se emplaza en la cuenca del río Guarapiche, ubicada en la región Nor-oriental de Venezuela; cubre parte del noroeste y noreste del estado Monagas, donde se encuentran los municipios Acosta, Cedeño y Maturín (Figura 1).

En esta cuenca se destacan las poblaciones agrícolas de Miraflores, Tristé, San Félix de Caicara, Merecure, Jusepín, Candelaria, San Vicente, La Cruz, Bajo Guarapiche, Plantación y Vuelta Larga; que son representativas del nivel socio productivo del estado (MARNR, 1980) y alto crecimiento demográfico y por la intervención de la actividad petrolera.

El río Guarapiche presenta una longitud de aproximadamente $238,04 \mathrm{~km}$ y forma parte de la Región Hidrográfica Oriental de Venezuela (Ministerio del Ambiente, 2006). A lo largo de su cauce principal se ubicaron las siete estaciones de muestreo (Figura 2). En el Tabla 1 se observan las coordenadas geográficas y UTM de las estaciones de muestreo.

Tabla 1. Coordenadas geográficas de las estaciones de muestreo en el río Guarapiche, Estado Monagas, Venezuela. Período febrero - julio 2011

\begin{tabular}{ccccc}
\hline Estación & Localidad & $\begin{array}{c}\text { Altitud Coordenadas } \\
\text { (m) }\end{array}$ & Geográficas \\
\hline 1 & Miraflores & 524 & $10^{\circ} 10^{\prime} 46^{\prime \prime}$ & $63^{\circ} 42^{\prime} 0^{\prime \prime}$ \\
2 & Tristé & 471 & $10^{\circ} 09^{\prime} 22^{\prime \prime}$ & $63^{\circ} 42^{\prime} 27^{\prime \prime}$ \\
3 & San Félix de Caicara & 235 & $09^{\circ} 57^{\prime} 35^{\prime \prime}$ & $63^{\circ} 39^{\prime} 31^{\prime \prime}$ \\
4 & Merecure & 110 & $09^{\circ} 46^{\prime} 18^{\prime \prime}$ & $63^{\circ} 33^{\prime} 54^{\prime \prime}$ \\
5 & Jusepín & 80 & $09^{\circ} 10^{\prime} 47^{\prime \prime}$ & $63^{\circ} 28^{\prime} 33^{\prime \prime}$ \\
6 & Bajo Guarapiche & 33 & $09^{\circ} 45^{\prime} 34^{\prime \prime}$ & $63^{\circ} 11^{\prime} 0^{\prime \prime}$ \\
7 & Palmonagas & 12 & $09^{\circ} 52^{\prime} 08^{\prime \prime}$ & $63^{\circ} 03^{\prime} 57^{\prime \prime}$ \\
\hline
\end{tabular}

Datos extraídos del dispositivo GPS modelo Magellan, 2011. Datum Regven

Las muestras de aguas se recolectaron en los meses de febrero a julio del año 2011. Las estaciones de muestreo se ubicaron en puntos cercanos a las comunidades que se abastecen del río. En cada estación de muestreo se realizaron medidas in situ de temperatura del agua, $\mathrm{pH}$ y conductividad eléctrica. Para ello se utilizó un sistema 


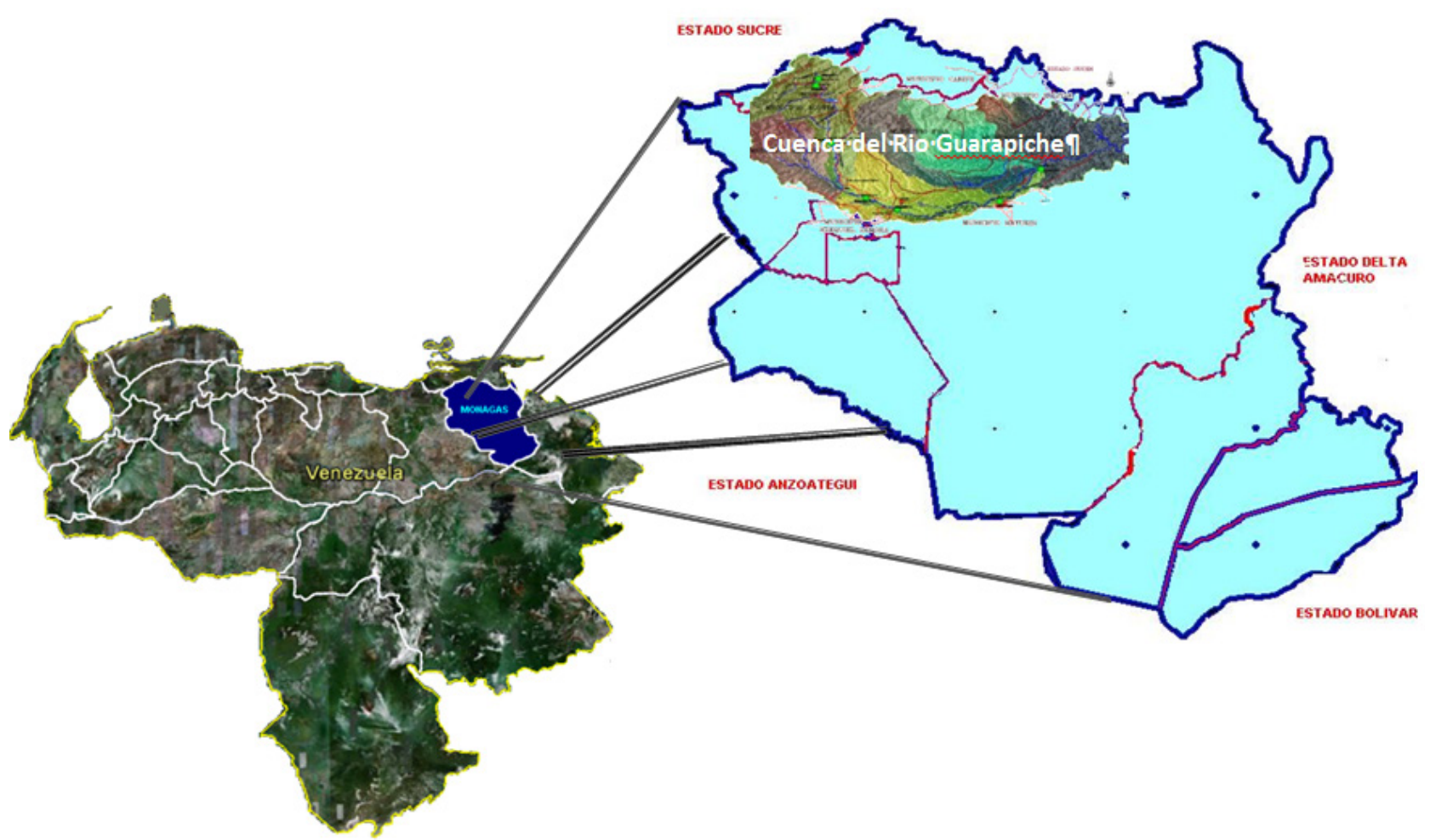

Figura 1. Ubicación del área de estudio

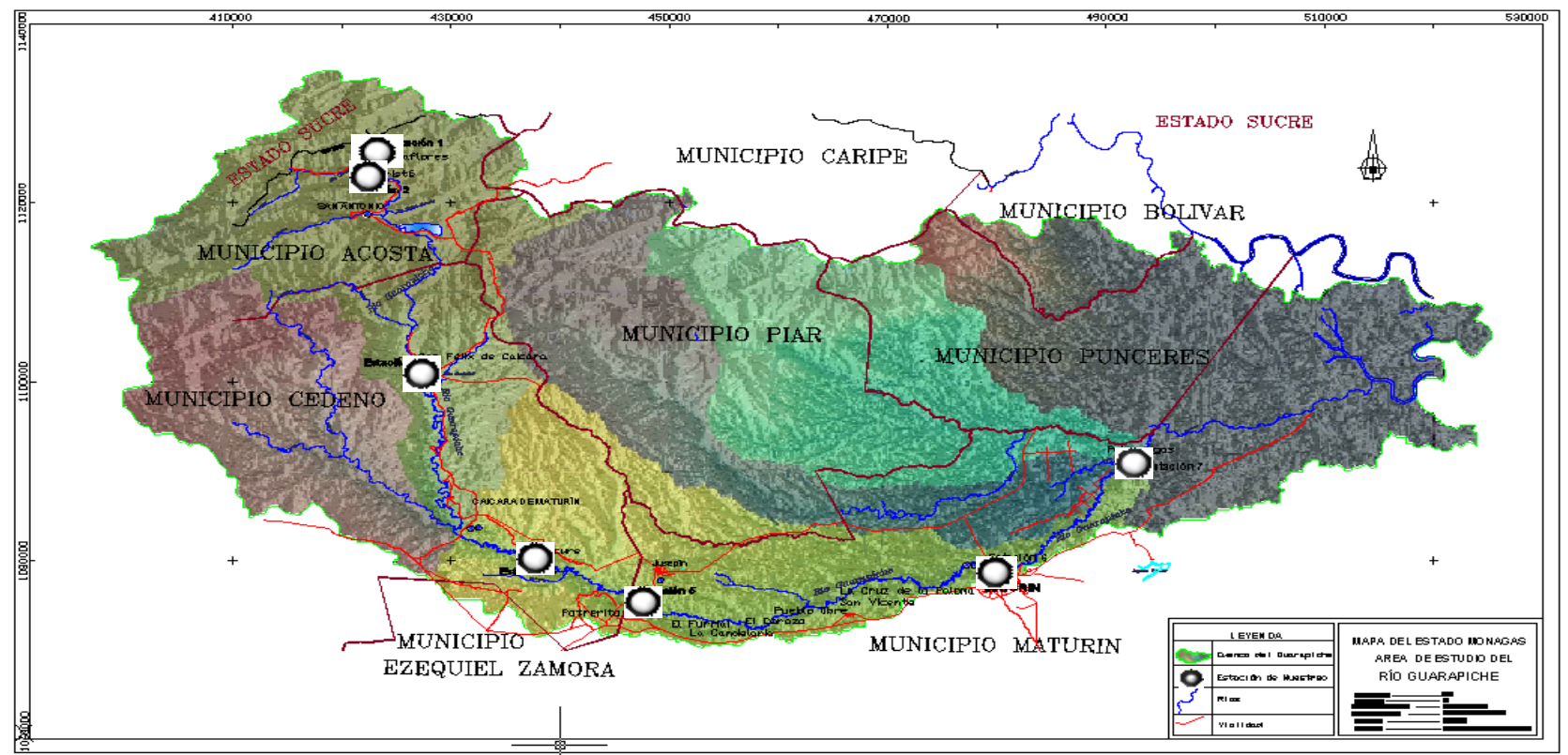

Figura 2. Ubicación de las estaciones de muestreo. Río Guarapiche, Monagas, Venezuela. Período febrero-julio 2011

multiparamétrico portátil conectado a un multisonda modelo T82 marca Hanna instruments. Las medidas de coordenadas geográficas y altitud se determinaron mediante un GPS modelo explorist 600 marca Magellan.

Las muestras se tomaron del centro del río, donde el flujo era más estable. Estas se captaron en envases plásticos de dos litros para los parámetros fisicoquímicos y de 0.25 litros para las variables microbiológicas, esterilizados de acuerdo a la metodología recomendada para la recolección de muestras químicas y de organismos microbiológicos (López et al., 1993). La toma de muestras, el método de conservación y los análisis para la determinación de las diferentes parámetros se ejecutaron de acuerdo a Standard methods for the examination of water and wastewater (APHA, 1995).

En el sitio de muestreo se sumergió el envase tapado dentro del agua hasta una profundidad de 15 a $30 \mathrm{~cm}$, se destapó y luego se volvió a colocar su tapa dentro del agua. La toma de muestra se efectuó con la boca del frasco en contracorriente, tratando de no dejar espacios de aire. Las muestras de aguas crudas se colocaron en una cava con hielo para mantenerlas a una temperatura de $4{ }^{\circ} \mathrm{C}$ hasta su destino final, para los análisis respectivos (Méndez et al., 2010). 


\section{Índice de calidad del agua (ICA)}

El ICA, se calculó en función de la idoneidad del agua subterránea para consumo humano consumo. Para determinar el ICA se siguieron tres pasos (Tiwari y Mishra, 1985; Singh, 1992). En el primer paso, se asignó un peso (wi) a cada uno de los catorce parámetros (temperatura, $\mathrm{pH}$, dureza, $\mathrm{CE}$, nitrato, nitritos, sulfato, cloruros, $\mathrm{OD}, \mathrm{Fe}$, $\mathrm{Mn}, \mathrm{Na}, \mathrm{K}$ y, CF) de calidad del agua de acuerdo con su relativa importancia en la calidad general del agua para consumo humano. Los valores asignados estuvieron en el rango de 1-4. Se asignó mucho peso a los parámetros que tiene efectos críticos para la salud y cuya presencia por encima de ciertos límites de concentración crítica podrían limitar el uso doméstico del recurso (Yidana et al., 2010). El peso máximo de 4 ha sido asignado a los parámetros como OD, Mn y CF debido a su mayor importancia en la evaluación de la calidad del agua (Srinivasamoorthy et al., 2008). El peso mínimo de 1 tiene ha sido asignado a los parámetros temperatura, dureza, potasio y sodio, debido a la menor importancia en la evaluación de la calidad del agua. Otros parámetros como $\mathrm{pH}$, conductividad eléctrica, dureza, nitritos, nitratos, sulfatos y cloruros se les asignaron un peso entre 1 y 4 dependiendo sobre su importancia en la determinación de la calidad del agua Tabla 2). Entonces, el peso relativo (RW) se calcula mediante la ecuación (1) de la siguiente manera (Singh, 1992):

$$
R W=\frac{W i}{\sum_{i=1}^{n} W i}
$$

Donde,

$\mathrm{RW}=$ el peso relativo,

$\mathrm{Wi}=$ el peso asignado de cada parámetro, $\mathrm{n}=$ el número de parámetros.

Los valores de peso relativo calculados (RW) de cada parámetro se dan en el Tabla 2.

Tabla 2. Peso relativo de los parámetros de calidad del agua

\begin{tabular}{ccccc}
\hline Parámetros & Unidades & $\begin{array}{c}\text { Estándar de } \\
\text { Calidad } \\
\text { del agua }\end{array}$ & $\begin{array}{c}\text { Peso } \\
\text { Asignado } \\
\text { Wi }\end{array}$ & $\begin{array}{c}\text { Peso } \\
\text { Relativo } \\
\text { RW }\end{array}$ \\
\hline $\mathrm{pH}$ & $\mathrm{pH}$ & $6.5-8.5(8.0)$ & 2 & 0.063 \\
$\mathrm{OD}$ & $\mathrm{mg} / 1$ & 4 & 4 & 0.126 \\
Temperatura & ${ }^{\circ} \mathrm{C}$ & 20 & 1 & 0.032 \\
Conductividad & $\mu \mathrm{S} / \mathrm{cm}$ & 3000 & 2.5 & 0.079 \\
Dureza & $\mathrm{mg} / 1$ & 500 & 1 & 0.032 \\
$\mathrm{~K}$ & $\mathrm{mg} / 1$ & 20 & 1 & 0.032 \\
$\mathrm{Na}$ & $\mathrm{mg} / 1$ & 200 & 1 & 0.032 \\
$\mathrm{Fe}$ & $\mathrm{mg} / 1$ & 1 & 3 & 0.095 \\
$\mathrm{Mn}$ & $\mathrm{mg} / 1$ & 0.1 & 4 & 0.126 \\
$\mathrm{NO}_{3}$ & $\mathrm{mg} / 1$ & 50 & 2.2 & 0.069 \\
$\mathrm{NO}_{2}$ & $\mathrm{mg} / 1$ & 3 & 2 & 0.063 \\
$\mathrm{SO}_{4}$ & $\mathrm{mg} / 1$ & 400 & 2 & 0.063 \\
$\mathrm{CF}$ & $\mathrm{NMP}$ & 100 & 4 & 0.126 \\
$\mathrm{Cl}$ & $\mathrm{mg} / 1$ & 600 & 2 & 0.063 \\
\hline $\mathrm{Total}$ & & & 31.7 & 1 \\
\hline
\end{tabular}

Se determinó una escala de calificación de calidad (Qi) para todos los parámetros, excepto para el $\mathrm{pH}$ y OD; dividiendo su concentración en cada muestra de agua por su estándar respectivo de acuerdo su valor, según las directrices de la OMS (2006), o los estándares venezolanos para el agua potable enunciados en el Decreto 883 (1995), el resultado se multiplicó por 100.

$$
\mathrm{Q}=\left[\frac{C_{i}}{s_{i}}\right] \times 100
$$

Mientras, la calificación de calidad para pH o OD (Q pH, OD) se calculó sobre la base de

$$
Q_{p H, D O}=\left[\frac{C_{i}-V_{i}}{S_{i}-V_{i}}\right] \times 100 \quad \ldots \ldots \ldots
$$

Donde:

$\mathrm{Qi}=$ la calificación de calidad.

$\mathrm{Ci}=$ concentración de cada parámetro químico en cada muestra de agua en $\mathrm{mg} / \mathrm{l}$.

$\mathrm{Si}=$ valor del parámetro de calidad del agua obtenido de la OMS recomendada o estándar venezolano para el agua potable enunciados en el Decreto 883 del parámetro correspondiente, en $\mathrm{mg} / 1$.

$\mathrm{Vi}=$ el valor ideal que se considera como 7.0 para $\mathrm{pH}$ y 14.6 para DO.

Las ecuaciones (2) y (3) aseguran que $\mathrm{Qi}=0$ cuando un contaminante está totalmente ausente en la muestra de agua y $\mathrm{Qi}=100$ cuando el valor de este parámetro es justo igual a su valor permisible. Por lo tanto, cuanto mayor es el valor de Qi, más contaminada está el agua (Mohanty, 2004). En el tercer paso, se calcula el subíndice (SIi) del i-ésimo parámetro con la Ecuación (4) para cada parámetro químico, luego se usa para calcular el ICA del índice de calidad del agua en general (Tiwari \& Mishra, 1985).

$$
S I i=R W \times Q i
$$

El ICA se calcula sumando los valores de cada subíndice de cada muestra de agua de la siguiente manera:

$$
I C A=\sum_{i=1}^{n} S I i
$$

Los valores de ICA calculados se clasificaron de acuerdo con Ramakrishnaiah et al. (2009) como excelente, bueno; pobre; muy pobre e Inadecuado o no apta para consumo humano (Tabla 3 ).

Un análisis estadístico se realizó a través de un ANOVA (Análisis de Varianza) al 5\% de probabilidad, utilizando el programa estadístico SAS (9.0), mostrando diferencias significativas entre los tratamientos, se aplicó una prueba de Duncan al 5\% de significancia. El análisis se realizó para detectar las variaciones de los parámetros a través del tiempo y en cada estación. También se determinaron las medidas de tendencias central y medidas de dispersión en un gráfico tipo caja de ploteo o box plot. Todos estos cálculos se llevaron a cabo con el paquete estadístico descriptivo SPSS (Versión 8). 
Tabla 3. Clasificación de las aguas de acuerdo con el ICA

\begin{tabular}{ccc}
\hline Valor del ICA & Clase & Calidad del Agua \\
\hline$<50$ & I & Excelente \\
$50-100$ & II & Buena \\
$100-200$ & III & Pobre \\
$200-300$ & IV & Muy Pobre \\
$>300$ & V & No apta para consumo \\
& & humano \\
\hline
\end{tabular}

Fuente: Ramakrishnaiah et al., 2009

\section{Resultados y discusión}

Las estadísticas descriptivas relacionadas con la calidad del agua se presentan en el Tabla 4. Los estadísticos descriptivos relacionados con los valores de calidad del agua de $\mathrm{CF}$, Dureza, $\mathrm{Cl}$ y $\mathrm{CE}$ parecen ser de distribución estándar muy alta. Estas variables cambian considerablemente en el río Guarapiche con respecto a su valor promedio, pero no influyen en la calidad de sus aguas porque no sobrepasan los valores límites de las normas venezolanas ni internacionales.

\section{Parámetros físicos}

Los parámetros físicos estudiados se detectaron con valores bajos que no exceden los límites permisibles para contaminantes en descargas de aguas y bienes nacionales, Norma Oficial Venezolana (Decreto 883, 1995). La temperatura es una de los parámetros más importante para un sistema fluvial. La variación en la temperatura del agua superficial afecta la solubilidad de sales, contenido de OD, tasa de biodegradación orgánica de los materiales y otros parámetros físico-químicos (Rao y Rao, 2010). Los valores de temperatura en el presente estudio variaron de $21.1^{\circ} \mathrm{C}$ (Estación Miraflores) a $29.6{ }^{\circ} \mathrm{C}$ (Estación Palmonagas). Los valores de $\mathrm{pH}$ presentaron un valor mínimo de 7.5 (Estación Palmonagas) y uno máximo de 8.4 (Estación Miraflores), que le da al agua del río una naturaleza ligeramente alcalina. Los valores de $\mathrm{pH}$ por encima de 8 observados en las tres primeras estaciones de la cuenca alta se deben posiblemente a las condiciones geológicas por la formación cretácea de la zona. La conductividad eléctrica en el agua se debe a la ionización de sólidos inorgánicos disueltos y se convierten en una medida de STD. Es el índice básico para verificar la idoneidad del agua para fines agrícolas (Rizvi et al., 2016). Los valores de CE en este estudio varían de $218 \mu \mathrm{S} / \mathrm{cm}$ (Palmonagas) a 445.08 $\mu \mathrm{S} / \mathrm{cm}$ (Triste y Jusepín). Mayor conductividad del agua corresponde a mezcla de las corrientes del agua del río.

\section{Parámetros químicos}

De los cationes estudiados (Fe, Mn, Na y K), el Fe y Mn en algunas estaciones de muestreo presentaron valores por encima de los estándares actuales de la Norma venezolana. El Fe se origina de los sedimentos erosionados de la parte alta de la cuenca del río y que son arrastrados hacia los cuerpos de agua a través de procesos de escorrentía hacia la cuenca baja. Los valores de Fe en el presente estudio variaron de $0 \mathrm{mg} / 1$ (Estación Miraflores) a 2.55 $\mathrm{mg} / \mathrm{l}$ (Estación Palmonagas).La presencia de Mn puede derivarse de la generación de procesos de reducción (bajo potencial redox) en las corrientes contaminadas con aguas residuales; estos procesos solubilizan metales como el Mn (Chávez-Alcántar et al., 2011). Las aguas residuales son también fuente de metales pesados (Chávez-Alcántar et al., 2011). Los valores de Mn presentaron un valor mínimo de $0 \mathrm{mg} / \mathrm{l}$ (Estación Miraflores) y uno máximo de 4.6 (Estaciones Triste y San Félix). En el caso de los aniones $\left(\mathrm{NO}_{2}, \mathrm{NO}_{3}, \mathrm{SO}_{4}, \mathrm{CL}\right.$ y $\left.\mathrm{OD}\right), \mathrm{NO}_{2}$ y $\mathrm{NO}_{3}$ presentaron valores por encima de la norma y el OD presentó valores por debajo de la misma.El ion nitrito $\left(\mathrm{NO}_{2}^{-}\right)$puede estar presente en las aguas bien como consecuencia de la oxidación del $\mathrm{NH}_{3}$ o como resultados de la reducción de los nitratos $\left(\mathrm{NO}_{3}^{-}\right)$. Su presencia en el agua puede ser una evidencia de contaminación reciente, dada su inestabilidad.

Tabla 4. Estadísticos descriptivos básicos de los parámetros físicos, químicos y biológicos estudiados en el río Guarapiche. Período febrero - julio, 2011

\begin{tabular}{|c|c|c|c|c|c|c|c|}
\hline \multirow[b]{2}{*}{ Parámetro } & \multirow[b]{2}{*}{ Unidad } & \multicolumn{2}{|c|}{ Rango(Nº Estación) } & \multirow[b]{2}{*}{ Media } & \multicolumn{2}{|c|}{ LMP } & \multirow{2}{*}{$\begin{array}{c}\text { Desviación } \\
\text { Estándar }\end{array}$} \\
\hline & & Mínimo & Máximo & & Decreto $883^{*}$ & OMS & \\
\hline Temperatura & ${ }^{\circ} \mathrm{C}$ & $21.1(1)$ & $29.6(7)$ & 26.31 & & & 2.76 \\
\hline $\mathrm{pH}$ & & $7.5(7)$ & $8.41(1)$ & 8.02 & $6.0-8.5$ & & 0.27 \\
\hline Dureza & $\mathrm{mg} / \mathrm{l}$ & $270.29(6)$ & $445.08(2,4)$ & 344.14 & 500.0 & & 47.34 \\
\hline $\mathrm{CE}$ & $\mu \mathrm{S} / \mathrm{cm}$ & $218(7)$ & $380(2,5)$ & 312.12 & & 3000 & 36.59 \\
\hline $\mathrm{Fe}$ & $\mathrm{mg} / \mathrm{l}$ & $0(1)$ & $2.55(7)$ & 0.38 & 1.0 & 0.3 & 0.51 \\
\hline $\mathrm{Mn}$ & $\mathrm{mg} / 1$ & $0(1,2)$ & $4.6(2,3)$ & 0.32 & 0.1 & 0.5 & 1.07 \\
\hline $\mathrm{Na}$ & $\mathrm{mg} / 1$ & $0(1,2,3,4)$ & $25.1(7)$ & 5.58 & 200 & & 8.60 \\
\hline $\mathrm{K}$ & $\mathrm{mg} / 1$ & $20(1,2)$ & 65.00 & $23.84(4)$ & & 50 & 7.89 \\
\hline $\mathrm{NO}_{2}$ & $\mathrm{mg} / 1$ & $0(1,2)$ & $14(2,4)$ & 0.83 & 5 & 3 & 2.40 \\
\hline $\mathrm{NO}_{3}$ & $\mathrm{mg} / 1$ & $0(1,2)$ & $13.2(4)$ & 0.87 & 5 & 50 & 2.48 \\
\hline $\mathrm{SO}_{4}$ & $\mathrm{mg} / 1$ & $0(1)$ & $40(4,5)$ & 23.51 & 400 & 250 & 8.71 \\
\hline $\mathrm{Cl}^{4}$ & $\mathrm{mg} / 1$ & $0(1)$ & $77(6,7)$ & 5.27 & 600 & 250 & 12.33 \\
\hline OD & $\mathrm{mg} / \mathrm{l}$ & $2.45(7)$ & $9.9(1)$ & 4.01 & $>3$ & & 3.09 \\
\hline $\mathrm{CF}$ & $\mathrm{NMP} / 100 \mathrm{ml}$ & $0(1)$ & $11000(6)$ & 496.21 & & & 1720.58 \\
\hline
\end{tabular}

LMP = límite máximo permitido por la norma venezolana. Decreto 883(1995)* y Organización Mundial de la Salud 
La toxicidad del ion nitrito determina la impotabilidad de la misma (Albert, 2009). De acuerdo con la legislación venezolana (Decreto 883), el agua para consumo humano debe tener niveles de nitratos + nitritos por debajo de 10 $\mathrm{mg} / \mathrm{l}$.

$\mathrm{El}$ promedio general de $\mathrm{NO}_{2}{ }^{-}+\mathrm{NO}_{3}{ }^{-}$observado en este estudio del río Guarapiche fue de 1,71 $\pm 3,89 \mathrm{mg} / \mathrm{l}$, con un valor máximo de 20 y un valor mínimo de $0 \mathrm{mg} / \mathrm{l}$. A lo largo del río Guarapiche, se observó que la estación que presentó los más altos niveles de nitratos y nitritos fue la estación 4 (Merecure), donde se observó valores de hasta $20 \mathrm{mg} / \mathrm{l}$, sobrepasando hasta en un $100 \%$ al valor umbral señalado en el Decreto 883 (1995). La zona de Merecure se caracteriza por ser una zona agrícola con alta actividad en la siembra de hortalizas y posiblemente una alta aplicación de fertilizantes nitrogenados, influya en los altos niveles observados en este estudio. También las lluvias pudieron haber arrastrado los residuos nitrogenados de dichas actividades y llevarlas al cauce del río, elevando así los niveles de nitratos y nitritos en esta estación. De la misma forma, concentraciones de nitritos serían indicadoras de condiciones anóxicas o de aportes contaminantes introducidos por líquidos residuales con presencia de nitrógeno amoniacal, como es el caso de los líquidos cloacales, contribuyendo esto a que se eleve los niveles bacteriológicos en estas estaciones (Gil et al., 2013). El oxígeno disuelto (OD), es la cantidad de oxigeno que está disuelto en el agua, esto se logra por la aireación y como producto de desecho de la fotosíntesis. La estación 6 (Maturín) recibe descargas de aguas negras de las plantas de tratamiento de las nuevas urbanizaciones que se desarrollan a lo largo de la cuenca baja del río Guarapiche, no obstante, tiene un nivel de OD mediano, indicando que posee capacidad para mantener vivos organismos aerobios y plantas. Las otras estaciones muestran niveles de OD dentro del límite establecido en el Decreto 883 (1995), por lo que el río cuenta con capacidad autodepurativa (Sperling, 2001). Solo el valor de OD en la estación 7 (Palmonagas) está fuera de la norma, indicando contaminación en ese punto e imposibilitando la supervivencia de la biota (Iriondo y Mota, 2004).

Dentro del balance gaseoso en los sistemas acuáticos, el oxígeno disuelto es fundamental, ya que es generado por los productores primarios y captado por el intercambio atmosférico por un lado y consumido por los organismos aeróbicos por el otro, los cuales lo utilizan para su síntesis energética, limitando o favoreciendo con la abundancia o escasez de este parámetro la presencia y/o ausencia de organismos acuáticos (Díaz et al., 2006).

\section{Parámetros microbiológicos}

Por último, para los CF la legislación venezolana emitida en el Decreto 883 (1995), el valor permisible de coliformes fecales para aguas de consumo humano debe ser 0 $\mathrm{NMP} / 100 \mathrm{ml}$ de agua y para el caso de uso agropecuario debe estar por debajo de 100NMP/100ml de agua. En esta investigación, se observó un valor promedio general de coliformes fecales de 496,21 $\pm 1720,57$, valor máximo de 11.000 y un valor mínimo de $0 \mathrm{NMP} / 100 \mathrm{ml}$. Valor que sobrepasa en casi un $500 \%$ al máximo valor permisible por la normativa vigente. Tomando en consideración las estaciones se observa que las que están por debajo del valor crítico son las estaciones 1,3 y 4 (Miraflores, San Félix y Merecure) y la más crítica con un valor promedio de 1963,33 NMP/100ml de coliformes fecales es la estación 6 de Maturín.

Actualmente en Venezuela, el problema de la contaminación microbiológica se presenta de manera generalizada, a causa de los vertimientos de aguas residuales en los ambientes marinos y costeros, así como también en otros ecosistemas acuáticos; problemática que se ve magnificada si se tiene en cuenta la falta de tratamiento de los desechos líquidos y sólidos a lo largo de la geografía nacional. De la calidad de las aguas depende en gran medida la supervivencia de las especies y de los ecosistemas, que a su vez reflejan la conservación o el deterioro en que se encuentran por causa de actividades o fenómenos tanto naturales como de tipo antrópicos, por lo tanto, es importante cumplir con las directrices y normativas que existen para garantizar una buena calidad del agua y por ende del ecosistema, y de los usos que de este se deriven (Gil et al., 2013).

\section{Índice de calidad del agua}

Los valores de ICA calculados para 7 estaciones en la cuenca del río Guarapiche variaron de 44.38 a 363.79 como se muestra en la Figura 3. El valor promedio observado fue de 83.10 lo que califica en forma general a las aguas en un nivel II, como aguas de buena calidad. La Tabla 5 muestra el porcentaje de muestras de agua que caen bajo las diferentes categorías de calidad. Por lo tanto, el 14.29 $\%$ del agua del río se ubicó en la clase I: agua excelente, el $69.05 \%$ en clase II - agua buena, el $11.90 \%$ en clase III pobre, el $2.38 \%$ en clase IV muy pobre y solo el $2.38 \%$ en clase $\mathrm{V}$ no apta para consumo humano. Si se toma el valor de ICA de 100, como límite superior o umbral para agua potable, podemos decir $83.34 \%$ (suma de las clases I y II), son aptas para consumo humano. Dos por ciento de las muestras de agua del río tomadas presentaron valores de ICA dos veces por encima del umbral de potabilidad señalado. La excelente calidad del agua pertenece a la zona de la cuenca alta donde nace el río. En cambio las aguas tipo IV y V se ubican en las estaciones 6 y 7, ubicadas en la cuenca baja, donde los altos valores de ICA observados son productos de las actividades antrópicas, entre las que destacan las actividades agrícolas, industriales petroleras y poblaciones, con sus respetivos efectos secundarios de explotación mineral y maderera, que resultan en el aumento de los valores de algunos parámetros químicos y microbiológicos. Los valores altos del ICA observados en las estaciones de Maturín y Palmonagas se encontraron que proviene principalmente de altos valores de los parámetros $\mathrm{Fe}, \mathrm{Mn}$, nitritos, nitratos, y $\mathrm{CF}$, con niveles superiores a los indicados en la normativa venezolana 
del Decreto 883 (1995). El análisis de varianza para la comparación de valores ICA según el mes y la estación, mostró diferencias significativas $(\mathrm{P}<0,05)$ para los meses y las estaciones. Tomando en consideración las estaciones y considerando como valor critico el valor de 100,( por encima se considera un agua de calidad pobre); se observa que las primeras seis estaciones se encuentran por debajo de este valor crítico y solo en la Estación de Palmonagas se observan la mayoría de las observaciones por encima de 100 (Figura 4).En la Figura 5 se observa que el mes más crítico con valores por encima de 100 es el mes de mayo, que coincide con el comienzo del período lluvioso, que favorece la proliferación de microorganismos. Este aumento puede ser causado por el incremento del caudal y escorrentía producido por las lluvias. Otra causa es la ausencia de plantas de tratamiento de aguas servidas en los asentamientos adyacentes al río donde se observan el uso de pozos sépticos o hay un vertido directo de desechos al río.

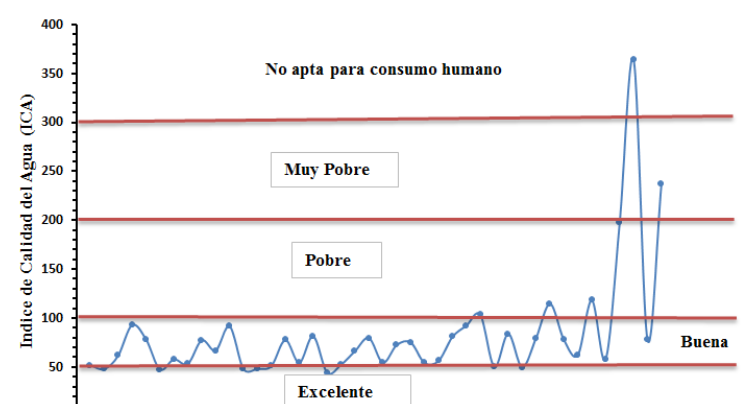

Figura 3. Variación de los valores del ICA en las muestras de agua estudiadas en el río Guarapiche, durante el período febrero-julio. 2011

Gil et al. (2013) y Rondón (2008), señalan que la calidad del agua del río Guarapiche estará muy vinculada al crecimiento demográfico de los centros poblados localizados en el valle. Incurriendo esto al aumento de todas las actividades que afectan de una manera u otra la calidad del agua sobre la actividad agrícola y mal funcionamiento de las plantas de tratamiento de aguas residuales.

Tabla 5. Clasificación de la calidad del agua de acuerdo al valor del índice de calidad del agua

\begin{tabular}{cccc}
\hline $\begin{array}{c}\text { Valor del } \\
\text { ICA }\end{array}$ & Clase & Calidad del Agua & $\begin{array}{c}\text { \% de la } \\
\text { Muestra }\end{array}$ \\
\hline$<50$ & I & Excelente & 14.29 \\
$50-100$ & II & Buena & 71.42 \\
$100-200$ & III & Pobre & 11.9 \\
$200-300$ & IV & Muy Pobre & 2.38 \\
$>300$ & V & No apta para consumo & 2.38 \\
& \multicolumn{3}{c}{ humano } \\
\hline
\end{tabular}

Durante la última década, el río Guarapiche ha sido sometido a un rápido declive en el estado de la calidad del agua que es posiblemente debido al aumento en la población y actividades humanas, tal como lo reporta Gil et al. (2013). Este efecto fue bastante evidente con estos resultados y puede implicar que las medidas preventivas adoptadas por las autoridades locales aún no son suficientes; está claro que la descarga doméstica y las actividades agrícolas, además de los efectos de la sequía de los últimos dos años son las principales amenazas para la calidad del agua del río Guarapiche. El estudio concluye que el ICA es una de las mejores herramientas para calcular el potencial de contaminación de manera comprensiva y que también se puede utilizar para clasificar la calidad del agua de una forma fácil de entender para todo criterio científico de calidad del agua.

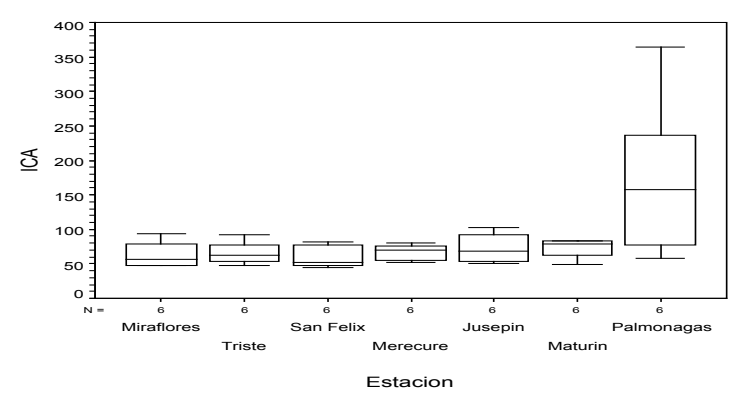

Figura 4. Cajas de ploteo para la variable ICA, observada en el río Guarapiche. Periodo febrero - julio 2011 Variación espacial

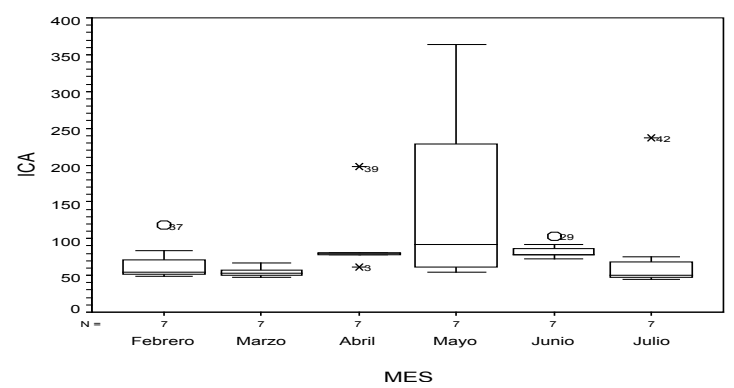

Figura 5. Cajas de ploteo para la variabe coliformes fecales, observada en el río Guarapiche. Período febrero julio 2011. Variación temporal

Un estudio regular del ICA puede servir de guía a las autoridades locales en la toma de decisiones adecuadas para implementar las medidas correctivas. Finalmente, se concluye que el río Guarapiche en su cuenca baja está al borde de la degradación y que se debe tomar medidas preventivas por parte de las autoridades competentes.

\section{Conclusiones}

Los parámetros físicos estudiados estuvieron dentro de los límites establecidas por las normas consideradas. Pero, de los cationes estudiados (Fe, Mn, Na y K), el Fe y Mn en algunas estaciones de muestreo presentaron valores por encima de los estándares actuales de la Norma venezolana. El NO${ }_{2}$ y NO$_{3}$ presentaron valores por encima de la norma y el OD presentó valores por debajo de la misma. En algunas estaciones se observaron valores de CF que sobrepasan en casi un $500 \%$ al máximo valor permisible por la normativa vigente. 
Los valores de ICA, van desde 44.38 (muestreo estación San Félix) a 363.69 (estación de muestreo Palmonagas). Los valores altos del ICA observados en las estaciones de Maturín y Palmonagas se encontraron que proviene principalmente de altos valores de los parámetros $\mathrm{Fe}, \mathrm{Mn}$, nitritos, nitratos, y $\mathrm{CF}$, con niveles superiores a los indicados en la normativa venezolana del decreto 883 .

Se concluye que las aguas del río Guarapiche en el área de su cuenca media y baja requieren algún tratamiento previo antes del consumo humano.

\section{Literatura citada}

Albert, L. 2009. Nitratos y nitritos. Recuperado de: http:// www.bvsde.ops-oms.org/bvstox/fulltext/toxico/ toxico-03a17.pdf.

American Public Health Association [APHA]. 1995. American Water Works Association (AWWA), Water Pollution Control Federation (WPCF) . Standard Methods for the Examination of Water and Wastewater, 19 ed. Washington, D.C.Arias, F. (2006). El Proyecto de Investigación, Introducción a la Metodología Cientifica $5^{\circ}$ Edición. Caracas - Venezuela: Espíteme C.A.

Bhattacharya, T.; Tuck, N. and Chakraborty, S. 2012. "Physicochemical analysis of groundwater quality of Anand district", ISCA Int. Res. J. Environ. Sci. $1(1) 33: 38$.

Canter, L.W. 1996. Environmental Impact Assessment, McGraw-Hill Series in Water Resources and Environmental Engineering, ISBN 0-07-009767-4, p. 122-133.

Chávez-Alcántar, A.1.; Velázquez-Machuca, M.; PimentelEquihua, J. L.; Venegas-González, J.; Montañez-Soto, J. L. y Vázquez-Gálvez, G. 2011. Hidroquimica de las aguas superficiales de la Ciénega de Chapala e índice de calidad de agua. Terra Latinoamericana 29 (1):13.

Cude, C. 2001. "A tool for evaluating water quality management effectiveness" $J$. American Water Resource. Assess 37:125-137.

Decreto $\mathrm{N}^{\circ} 883$. Normas para la clasificación y el control de la calidad de los cuerpos de agua y vertidos o efluentes líquidos. Gaceta Oficial de la República de Venezuela, № 5.021 Extraordinaríos del 18 de diciembre de 1995

Díaz R. S.; Aguirre L. y Cano Q. 2006. Evaluación ecológica de las comunidades de peces en dos sistemas lagunares estuarinos del sur de Chiapas, México. Hidrobiológica 16 (2): 197-210.

Gil, M. J. A.; Belloso de Herrera, G.; Vizcaino, G. C.; Maza, I.J.; Sánchez, C.M.C.; Bolívar, C.E. y Martínez, P.D. 2013. Evaluación de la calidad microbiológica y niveles de nitratos y nitritos en las aguas del río Guarapiche, estado Monagas, Venezuela. Revista Cientifica UDO 154 Agrícola 13(1): 154-163.

Iriondo, A. y Mota, J. 2004. Desarrollo de una red neuronal para estimar el oxígeno disuelto en el agua a partir de instrumentacion de EDAR. $X X V$ Jornadas de Automática.8-10 de Septiembre. Universidad de Castilla la Mancha.Ciudad Real, España.

Kung, H.; Ying, L. and Liu, Y. 1992. A Complementary Tool to Water Quality Indices: Fuzzy Clustering Analysis. Water Resour. Bull. 28(3):525-533.

Lekshmiprasad, S. and Mophin, K. 2017. Water Quality Assessmentof Ashtamudi Lake Using Nsfwqi .International Journal of Emerging Technology and Advanced Engineering Recuperado de: www.ijetae. com 7(1).

López, A.; Ruiz, D.; Hernández, G.; Fernández, E.; Mota, L.; Quiñones, E.; Rodríguez, R. 1993. Manual de laboratorio de microbiología sanitaria. Instituto Politécnico Nacional. Segunda edición.

Méndez, N. R.; San Pedro, C. L.; Castillo, B. E. y Vázquez, B. E. 2010. Modelación del tiempo de conservación de muestras biológicas de agua. Revista Internacional de Contaminación Ambiental 26(4).

Ministerio del Ambiente y de los Recursos No Renovables [MARNR]. 1980. Uso actual y perspectivas de aprovechamiento del río Guarapiche. Maturín: Serie informes técnicos Zona 12/IT. Maturín, Estado Monagas.

Ministerio del Ambiente. 2006. Recursos hídricos de Venezuela. Caracas. Fundambiente.

Mohanty, S.K. 2004. Water Quality Index of Four Religious Ponds and its Seasonal Variation in the Temple City,Bhuvaneshwar," In: A. Kumar, Ed., Water pollution, APH Publishing Corporation, New Delhi. pp. 211-218.

Organización Mundial de la Salud [OMS]. 2006. Guías para la calidad del agua potable. Primer apéndice a la tercera edición. Volumen 1. Recomendaciones. Organización Mundial de la Salud, Genève 27, Suiza ; 393 pp.

Ramakrishnaiah, C. R.; Sadashivaiah, C. and Ranganna, G. 2009. Assessment of Water Quality Index for the Ground Waterin Tumkur Taluk. E-Journal of Chemistry 6(2):523-530.

Rao, G.S. and Rao, G.N. 2010. Study of ground water quality in greater Viskhapatnun city, Andhra Pradesh (India)," J. of Env. Sc. \& Engg. 52:137-146.

Rizvi, N.; Deeksha, K.; Varun, J. 2016. World Academy of Science, Engineering and Technology International Journal of Environmental, Chemical, Ecological, Geological and Geophysical Engineering 10(1):10.

Rondón, C. 2008. Análisis ambiental del Río Guarapiche como fuente de abastecimiento de agua de Maturín. Estado Monagas. Venezuela. (Trabajo especial de grado). Universidad de Margarita, Nueva Esparta

Sanchez, E.; Colmenarejo, M.F.; Vicente, J.; Rubio, A.; Garcia, M.G.; Travieso, L. and Borja, R. 2007. Use of the water quality index and dissolved oxygen deficit as simple indicators of watersheds pollution. Ecol. Indic. 
7(2):315-328.

Singh, DF, 1992. Studies on the water quality index of some major rivers of Pune, Maharashtra. Proceedings of the Academy of Environmental Biology 1(1):61-66.

Sperling, M.V .2001. Princípios Básicos do Tratamento de Esgotos. $4^{\mathrm{a}}$ ed. Universidade

Federal de Minas Gerais.Brasil. 211 pp.

Smith, D.G. 1990. A better water quality indexing system for rivers and streams. Water Res. 24(10): 1237-1244.

Srinivasamoorthy, K.; Chidambaram, M.; Prasanna, MV.; Vasanthavigar, M, John, P. and Anandhann P. 2008. Identification of major sources controlling groundwater chemistry from a hard rockTerrain - a case study from Mettur Taluk, Salem District,Tamilnadu India. J Earth Syst Sci. 117(1):49-58.

Tiwari, T.N. \& Mishra, M.A. 1985. A preliminary assignment of water quality index of major Indian rivers. Indian JEnviron Protect 5:276-279.

Yidana, SM.; Banoeng-Yakubo, B. and Akabzaa, TM. 2010. Analysis ofgroundwater quality using multivariate and spatial analyses inthe Keta basin Ghana. $J$ Afr Earth Sci 58(2):220-234. 